\title{
Millimeter VLBI detection of the TeV blazar Markarian 501
}

\author{
Marcello Giroletti*1 and Gabriele Giovannini ${ }^{1,2}$ \\ ${ }^{1}$ INAF Istituto di Radioastronomia, via Gobetti 101, 40129 Bologna, Italy \\ ${ }^{2}$ University of Bologna, Department of Astronomy, via Ranzani 1, 40127 Bologna, Italy \\ E-mail: giroletti@ira.inaf.it, ggiovann@ira.inaf.it
}

\begin{abstract}
We report on observations of the TeV blazar Mrk 501 with the Global mm-VLBI Array at 86 $\mathrm{GHz}$, using $3 \mathrm{C} 345$ as a calibrator. With the solutions from the calibrator and fringe fitting the data, we clearly detect a compact component even on transatlantic baselines yielding a resolution of $\sim 0.1 \times 0.2$ mas. A jet-like feature is also visible in the same position angle found in lower resolution images. This is one of the first mm-VLBI images of a TeV blazar and it provides information on the physics of the radio core on scales of a few hundreds Schwarzschild radii. Moreover, it is an encouraging starting point for future observations at higher frequencies and/or of weaker targets.
\end{abstract}

8th European VLBI Network Symposium

September 26-29, 2006

Toruń, Poland

\footnotetext{
${ }^{*}$ Speaker.
} 




Figure 1: Image of the calibrator 3C 345.

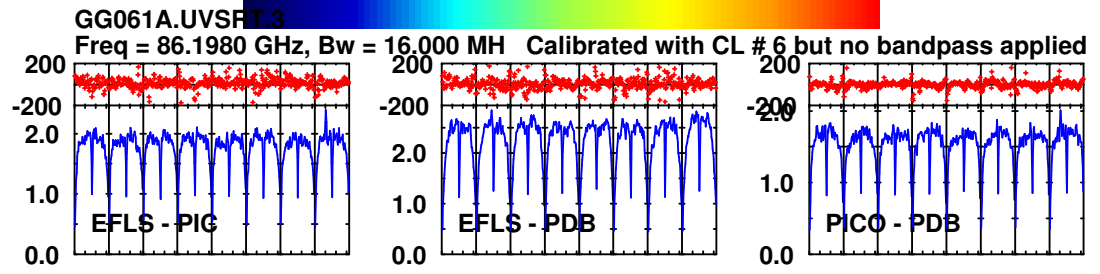

Figure 2: Phase and amplitude vs. frequency plots for a 5 min scan on Mrk 501. Baselines between Effelsberg, Pico Veleta and Plateau de Bure are shown.

\section{Introduction}

The BL Lac object Markarian 501 is a complex, core dominated radio source. Thanks to its proximity and brightness, the source is an ideal laboratory for experiments using advanced VLBI techniques: it is at $z=0.034$ ( 1 mas $=0.67 \mathrm{pc}$, using $H_{0}=70 \mathrm{~km} \mathrm{~s}^{-1} \mathrm{Mpc}^{-1}$ ), the Schwartzschild radius for its central black hole is estimated around $1 R_{S}=10^{-3} \mathrm{pc}$ (adopting $M_{B H}=10^{9} M_{\odot}$ ), and the total flux density at $5 \mathrm{GHz}$ is $S_{5}=1.4 \mathrm{Jy}$. We discussed some of its parsec scale properties in [1]. Now we extend our understanding of the source physics by going to the high resolution provided by the VLBI technique at the 3-mm band. Here we present preliminary results; they will be further discussed in a forthcoming paper.

\section{Global mm-VLBI observations}

We observed Mrk 501 on 14 Oct 2005 for 9 hours with the Global mm-VLBI Array [2]. The standard frequency was $86.453 \mathrm{GHz}$ and the participating telescopes were Effelsberg, Pico Veleta, Plateau de Bure, Onsala, Metsähovi, and 8 VLBA stations. This experiment tested the sensitivity limits of the array, since Mrk 501 was expected to be only a few hundreds mJy at this frequency. 


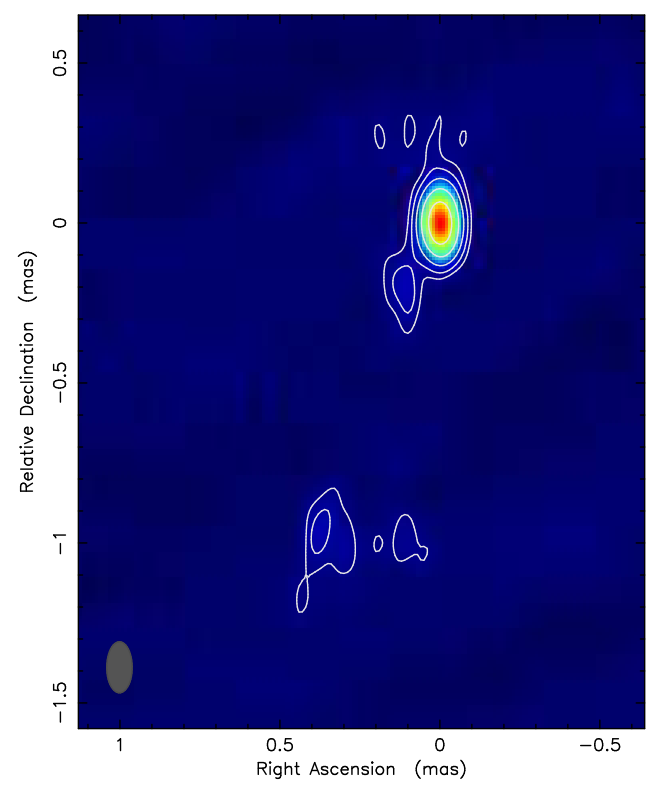

Figure 3: Preliminary map of Mrk 501 from the Global mm-VLBI Array.

The calibrator 3C345 was well detected (see Fig. 1). From the fringe fitting of 3C 345 we determined rates and delays, and applied them to the source. Fringe fitting of Mrk 501 itself with $\operatorname{APARM}(5)=2$ provided then good solutions on most baselines. The spectral plot in Fig. 2 shows the resulting data on some baselines before averaging in frequency.

In Fig. 3, we show our Global mm-VLBI Array image of Mrk 501. The beam FWHM is 160 $\mu$ as $\times 80 \mu$ as, and the image peak is $154 \mathrm{mJy} / \mathrm{beam}$. The lowest contours are traced at $\pm 4 \%$ of the peak, i.e. $\sim 6 \mathrm{mJy}$. We believe that it will be possible to improve the SNR over this preliminary result. The most remarkable features apparent so far are as follows:

1. The resolution is $\sim 560$ Schwarzschild radii; at this resolution, the core is still unresolved.

2. We detect some diffuse jet emission in P.A. $\sim 170^{\circ}$, in agreement with images at $22 \mathrm{GHz}$. The jet has therefore a still different orientation w.r.t. the ones at $>2\left(\right.$ jet $\mathrm{PA} \sim 100^{\circ}$ ) and $>20$ mas (jet $\mathrm{PA} \sim 45^{\circ}$ ) [1].

3. Given that such a weak source was detected, the performance of the existing mm-VLBI array is encouraging and future upgrades (e.g. new receivers at Plateau de Bure, inclusion of new or existing telescopes) promising.

4. A preliminary scientific outcome of this experiment is the study of the core spectrum: the flux density at $86 \mathrm{GHz}$ follows the optically thin part of the core spectrum measured at lower frequency [1].

We thank the personnel of the observatories participating in the Global mm-VLBI array and particularly T. Krichbaum for his advice during the data reduction.

\section{References}

[1] Giroletti M., Giovannini G., Feretti L., Cotton W.D., Edwards P.G., et al. 2004, ApJ 600, 127

[2] http://www.mpifr-bonn.mpg.de/div/vlbi/globalmm/ 